OPEN ACCESS

Edited by:

Chin Leong Lim,

Nanyang Technological University,

Singapore

Reviewed by:

Hamid Arazi,

University of Guilan, Iran

Christopher Latella,

Edith Cowan University, Australia

*Correspondence:

Ewan Thomas

ewan.thomas@unipa.it

Specialty section: This article was submitted to

Exercise Physiology,

a section of the journal

Frontiers in Physiology

Received: 02 December 2021

Accepted: 27 January 2022

Published: 28 February 2022

Citation:

Senna GW, Dantas EHM, Scudese E, Brandão PP, Lira VA, Baffi M,

Ribeiro LCP, Simão R, Thomas $E$ and

Bianco A (2022) Higher Muscle

Damage Triggered by Shorter

Inter-Set Rest Periods in Volume-

Equated Resistance Exercise.

Front. Physiol. 13:827847.

doi: 10.3389/fphys.2022.827847

\section{Higher Muscle Damage Triggered by Shorter Inter-Set Rest Periods in Volume-Equated Resistance Exercise}

\author{
Gilmar Weber Senna ${ }^{1,2}$, Estélio Henrique Martin Dantas ${ }^{2,3}$, Estevão Scudese ${ }^{1,2}$, \\ Paula Paraguassú Brandão' ${ }^{2}$, Vitor A. Lira ${ }^{4}$, Matheus Baffi', Luiz Claudio Pereira Ribeiro², \\ Roberto Simão ${ }^{5}$, Ewan Thomas ${ }^{6 *}$ and Antonino Bianco ${ }^{6}$
}

'Sports Science and Exercise Laboratory (LaCEE), Petrópolis Catholic University, Petrópolis, Brazil, ${ }^{2}$ Nursing and Biosciences Post-graduation Program, Doctorate of Federal University of State of Rio de Janeiro, Rio de Janeiro, Brazil, ${ }^{3}$ Biosciences Laboratory of Human Movement (LABIMH), Tiradentes University, Aracaju, Brazil, ${ }^{4}$ Department of Health and Human Physiology, The University of lowa, lowa City, IA, United States, ${ }^{5}$ School of Physical Education and Sports, Federal University of Rio de Janeiro, Rio de Janeiro, Brazil, ${ }^{6}$ Sport and Exercise Sciences Research Unit, Department of Psychology, Educational Science and Human Movement, University of Palermo, Palermo, Italy

Objectives: The aim of the manuscript was to analyze the effects of two rest periods between volume-equated resistance exercise (RE) on inflammatory responses (cytokines and leukocyte) and muscle damage.

Methods: Ten trained men $(26.40 \pm 4.73$ years, $80.71 \pm 8.95 \mathrm{~kg}$, and $176.03 \pm 6.11 \mathrm{~cm})$ voluntarily participated in training sessions consisting of five sets of 10 reps performed at 10-RM on (1) the barbell bench press followed by (2) leg press, with either 1- or 3-min rest between sets and exercises. Circulating concentrations of different biomarkers was measured before (Pre), and after $3 \mathrm{~h}$ (excepted for cytokines), 6, 12, and $24 \mathrm{~h}$ from exercise. The rate of perceived exertion (RPE) was recorded after each set on both planned visits.

Results: We found greater increases triggered by the 1-min rest period in Creatine Kinase (CK), occurring from 12 to $24 \mathrm{~h}$ post-exercise compared to the 3-min rest condition. A significant increase in the 1-min rest condition was also observed in the total number of leukocytes, neutrophils, and monocytes. The 1-min rest period also triggered increases compared to baseline in pro-inflammatory cytokines [Interleukin 1 beta (IL-1 $), p=0.004$; tumor necrosis factor $\alpha$ (TNF- $\alpha), p=0.01$; and granulocyte-macrophage colony-stimulating factor (GM-CSF), $p=0.01$ ], which were more evident after 6 and $12 \mathrm{~h}$ post-exercise. Similarly, increases in anti-inflammatory cytokines [Interleukin 5 (IL-5), $p=0.01$; Interleukin 6 (IL-6), $p=0.01$; and Interleukin 10 (IL-10), $p=0.01$ ] at all time-points were observed.

Conclusion: Our results indicate that a 1-min rest condition in volume-equated RE promoted greater overall muscle tissue damage with a longer duration of the inflammatory processes compared to a 3-min rest.

Keywords: cytokines, creatine kinase, L-Lactate Dehydrogenase, leukocytes, resistance training, physical fitness 


\section{INTRODUCTION}

Regarding general neuromuscular health, resistance exercise (RE) is considered the most efficient method for enhancing muscular strength, power, and muscular endurance (American College of Sports Medicine, 2009), triggering multiple benefits with numerous positive outcomes evident in the literature (Garber et al., 2011). However, the beneficial effects of resistance exercise can be influenced by exercise selection, exercise order, intensity, number of sets, and resting interval between sets (American College of Sports Medicine, 2009). Modulation of such parameters determines specific adaptations toward increases in strength or hypertrophy (Borde et al., 2015; Schoenfeld et al., 2019).

In particular, regarding modulation of inter-set lengths, evidence suggests that either shorter or longer inter-set duration (within a range of $60 \mathrm{~s}$ to $3 \mathrm{~min}$ ) may promote muscle hypertrophy (Grgic et al., 2017), while longer rests (usually $3 \mathrm{~min}$ or longer) seem to be more beneficial for strength development (de Salles et al., 2009). Therefore, it is still not clear which mechanisms mediated by inter-set length may determine hypertrophic adaptations. Previous studies indicated that differences in resting intervals between sets may independently influence the repetition performance (Senna et al., 2011, 2016; Scudese et al., 2015; Matos et al., 2021), as well as promote changes in neuromuscular (Willardson and Burkett, 2008), endocrine (Scudese et al., 2016), cardiorespiratory (Ratamess et al., 2007), and even immune responses to the exercise session (Gerosa-Neto et al., 2016; Rossi et al., 2016).

During RE sessions, the muscular tissue is exposed to microinjury (which occurs after concentric and eccentric contractions, with greater damage observed after eccentric exercise) (Markus et al., 2021). Exercise-induced muscle damage (EIMD) has been mainly observed in the sarcolemma, cytoskeleton, and contractile elements of the muscle. Despite EIMD may have short-term (24-48h) detrimental effects on muscle function, due to pain and inflammation, it is thought that the associated muscle inflammation promotes increased protein turnover, which may lead to long-term adaptations (Schoenfeld, 2012; Fukada et al., 2020). In particular, short-inter-set lengths (60 s or below) are seen to promote greater muscle damage than longer rest periods ( 3 min or above) (Machado and Willardson, 2010) and acutely increase anabolic hormonal levels (Henselmans and Schoenfeld, 2014). EIMD also increases the expression of both pro and anti-inflammatory cytokines of local muscle tissue (Calle and Fernandez, 2010; Minari and Thomatieli-Santos, 2021). Modulation of genetic muscle regulators and activation of muscle satellite cells, mediated by different anti-inflammatory cytokines, may be one of the major factors that lead to the regeneration or even hypertrophy of muscle tissue (Peake et al., 2006). However, muscle damage and inflammatory response derived from RE are still not completely elucidated and might play a key role regarding the physiological mechanism related to RE adaptations (Spiering et al., 2008; Margaritelis et al., 2021).

Research has indicated that once a tissue is damaged, similar increases in biochemical markers occur as a consequence of the extravasation of the content of the damaged cells, despite different stimuli are provided (Rodrigues et al., 2010; Evangelista et al.,
2011; Machado et al., 2012). Increases which also occur after acute bouts of high-intensity interval training [increases observed in Interleukin 6 (IL-6), Interleukin 10 (IL-10), and tumor necrosis factor $\alpha$ (TNF- $\alpha$ ); Gerosa-Neto et al., 2020]. After muscle damage occurs, an increase in leukocyte recruitment to the bloodstream is observed as an attempt to reverse the general breakdown (Kilgore et al., 2002; Fatouros and Jamurtas, 2016) due to the process of repair and tissue remodeling (Raastad et al., 1985).

Changes in the hemodynamic profile driven by mechanical stimuli, promote increased sympathetic action with release of catecholamines during exercise. These appear to be an important factor driving the elevation of leukocyte concentration, promoting the cellular recruitment of lymphoid organs and endothelium to the blood by mechanical loading (Pedersen and HoffmanGoetz, 2000).

Consequently, different cytokines have effects on the mobilization of neutrophils from the bone marrow to the blood, increasing the leukocyte count beyond the patterns presented immediately at the end of an exercise session (Pedersen and Hoffman-Goetz, 2000), thereby triggering a second phase increase in cell types. This physiological cascade of events that characterizes the immune system profile occurs at different time-point peaks that may not immediately fall after the exercise (Mayhew et al., 2005; Chatzinikolaou et al., 2010). Although the general inflammatory response is vastly clinically studied, little is known between the immune system response related to modulation of RE parameters, as different rest intervals between sets (Mayhew et al., 2005). Few experiments have attempted to elucidate the behavior of this process by analyzing the biomarkers of tissue damage, leukocyte profile, and cytokine expression of pro and anti-inflammatory action after RE performed with different intervals between sets (Rodrigues et al., 2010; Evangelista et al., 2011; Machado et al., 2012; Rossi et al., 2016). Therefore, the purpose of this study was to analyze the effects of two distinct rest period lengths (1 and $3 \mathrm{~min}$, which are typically used in hypertrophy or strength protocols, respectively) between RE sets of equalized volume on muscle tissue damage [CK and Lactate Dehydrogenase (LDH)] and inflammatory biomarkers responses.

\section{MATERIALS AND METHODS}

\section{Experimental Approach to the Problem}

Inter-set lengths represent a crucial aspect of modern resistance training. Different interest lengths are used in hypertrophy or strength training. In our study, inter-set length in volumeequated RE protocols represented the primary independent variable. Biomarkers of muscle damage and inflammation were dependent variables on the experimental time exposure. A cross-over research design was carried out due to the lack of a comparison/control group; therefore, intra-group comparisons were used between the two conditions.

\section{Subjects}

Ten trained men with at least 1 year of consistent RE experience were selected to participate $(26.40 \pm 4.73$ years, $80.71 \pm 8.95 \mathrm{~kg}$, 
$176.03 \pm 6.11 \mathrm{~cm}, 9.86 \pm 3.25 \%$ body fat, Bench Press (BP) relative strength: $1.27 \pm 0.27 \mathrm{~kg}^{\mathrm{kg}} \mathrm{kg}^{-1}$ of body mass, Leg press (LP) relative strength: $3.66 \mathrm{~kg} \cdot \mathrm{kg}^{-1}$ of body mass.). With the intent to standardize the subject selection, the following inclusion criteria were adopted: (a) engaged in at least 1 year of resistance training with a frequency of at least four times per week, with session duration approximately $1 \mathrm{~h}$; (b) non-usage of any ergogenic substance that would enhance repetition performance; (c) no acute or chronic injuries that would affect BP and LP performance; (d) no usage of medicines that may alter metabolism; (e) no pre-established endocrine diseases, cardiopathies, arterial hypertension, uncontrolled asthma, and any musculoskeletal conditions that could serve as an intervening factor in the practice of the activity (osteoarthritis, recent fracture, tendinitis, and use of prosthesis) and immune system alterations; and (f) agreement to not engage in any type of intense or structured physical activity throughout test days. Before data collection, all subjects answered "no" to all PAR-Q questions (Shephard, 1988). The study procedures were approved by the Federal University of State of Rio de Janeiro ethics committee (CAAE 63803717.2.0000.5285). Besides, all subjects read and signed a consent form after being informed of the testing procedures according to the Declaration of Helsinki.

\section{Ten Maximum Repetitions Test}

After two familiarization sessions, subjects completed the 10 maximum repetitions (10-RM) test sessions for bench press (BP) and leg press (LP), with each session separated by $72 \mathrm{~h}$. Each familiarization session consisted in simulating the testing protocols for both exercises (BP and LP) without the use of additional weight. For instance, participants were instructed on how they should perform exercise techniques with proper execution, in order to minimize the chance of injury, and they were also asked about their subjective rate of perceived effort intending to train their ratings when the actual testing sessions began. The 10-RM testing procedures followed the recommendations of Baechle and Earle (2000). To minimize testing errors, the following strategies were adopted for all exercise sessions: (a) standardized instructions were provided to each subject; (b) proper BP and LP technique was explained to all subjects, and compliance with this technique was verified for each repetition by an experienced investigator; (c) body positioning on the BP and LP was held constant and maintained throughout the whole experiment; and (d) verbal encouragement was applied during all testing sessions to encourage maximum effort from each subject; and $€$ the weight of the barbell and all plates were measured with a precision scale.

For the 10-RM determination, a progressive loading strategy was adopted, so that the minimum increase of weight was a total of $2 \mathrm{~kg}$ for BP, and $5 \mathrm{~kg}$ of LP after every successful attempt. After the warm-up, a maximum of five attempts was permitted for each 10-RM testing session, with a minimum of 10 -min recovery between the lifting attempts.

Excellent test-retest reliability was verified by the intraclass correlation coefficient (BP, $r=0.99$; $\mathrm{LP}, r=0.99$ ) for the 10-RM test. In addition, a paired student $t$ test did not show any significant differences between test-retest $1 \mathrm{RM}$ loads (BP, $p=0.16$; LP, $p=0.10$ ).

\section{Muscle Tissue Damage Biomarkers and Leukocyte Count}

All muscle tissue damage biomarkers and leukocyte count were verified at pre-exercise, $3,6,12$, and $24 \mathrm{~h}$ post-exercise, with the blood samples being collected by venipuncture from an antecubital vein for following the determination of the concentrations of circulating CK and LDH, Leucocyte counts and its sub-types. Biochemical analysis was performed using commercial kits [lots of the CK: 182906-01 (Roche); LDH: 00202541 (Roche); Reagent Cellpack: p7066; Stromatolyser 4DL: p6014; and Sulfolyser: p6009] specific to humans in an automatic device [Cobas E601 (Roche)]. For $\mathrm{CK}$ and $\mathrm{LDH}$, we used the electrochemiluminescence method. The hematological analysis was performed immediately after the collection through automated analysis (KX-21N, Sysmex) using the tubes containing the blood collected over the several distinct time-points by the photometric method.

\section{Cytokines Concentrations}

The cytokines levels for interleukin (IL)-1 $\beta$, IL-5, IL-6, IL-10, tumoral necrosis factor alfa (TNF- $\alpha$ ), and granulocytemacrophage colony-stimulating factor (GM-CSF) in the serum were determined by in an automatic device multiplex (Luminex ${ }^{\mathrm{TM}}$ 100/200 model) flow cytometry-based. The cytokine analysis was performed using commercial kits to magnetic cytokine human panel [Thermos-Fisher Scientific ${ }^{\mathrm{TM}}$ (LHC0001M)]. Assays performed by this system provide comparable intra- and interassay precision with typical coefficient of variation values of $<10 \%$ over the analyzed concentration range. Blood samples were collected at Pre, 6, 12, and $24 \mathrm{~h}$ post-exercise for this analysis. All samples for each participant were collected considering the timing of the workout session. After data collection, the IL-10/TNF- $\alpha$ ratio was calculated. For all assessments, the analytical sensitivity was $<0.5 \mathrm{pg} . / \mathrm{ml}$; except for GM-CSF that the analytical sensitivity was $<0.05 \mathrm{pg} . / \mathrm{ml}$. A 1:3 dilution between the curve points was adopted for this experiment.

\section{Rate of Perceived Exertion Procedure}

The OMNI RE scale (Lagally and Robertson, 2006) was implemented to obtain the (RPE) values. Subjects were previously familiarized and asked to choose a number based on their perceived exertion or subjective intensity of effort, strain, discomfort, and fatigue experienced during the exercise session. Immediately after each exercise set, subjects were asked to identify their RPE to provide a subjective measure of the exertion level.

\section{Resistance Exercise Session}

One week after the last 10-RM test session, subjects completed the first workout session, which consisted of the execution of the BP and subsequently the LP exercise. The starting order of inter-set length conditions (1- or 3-min rest between 


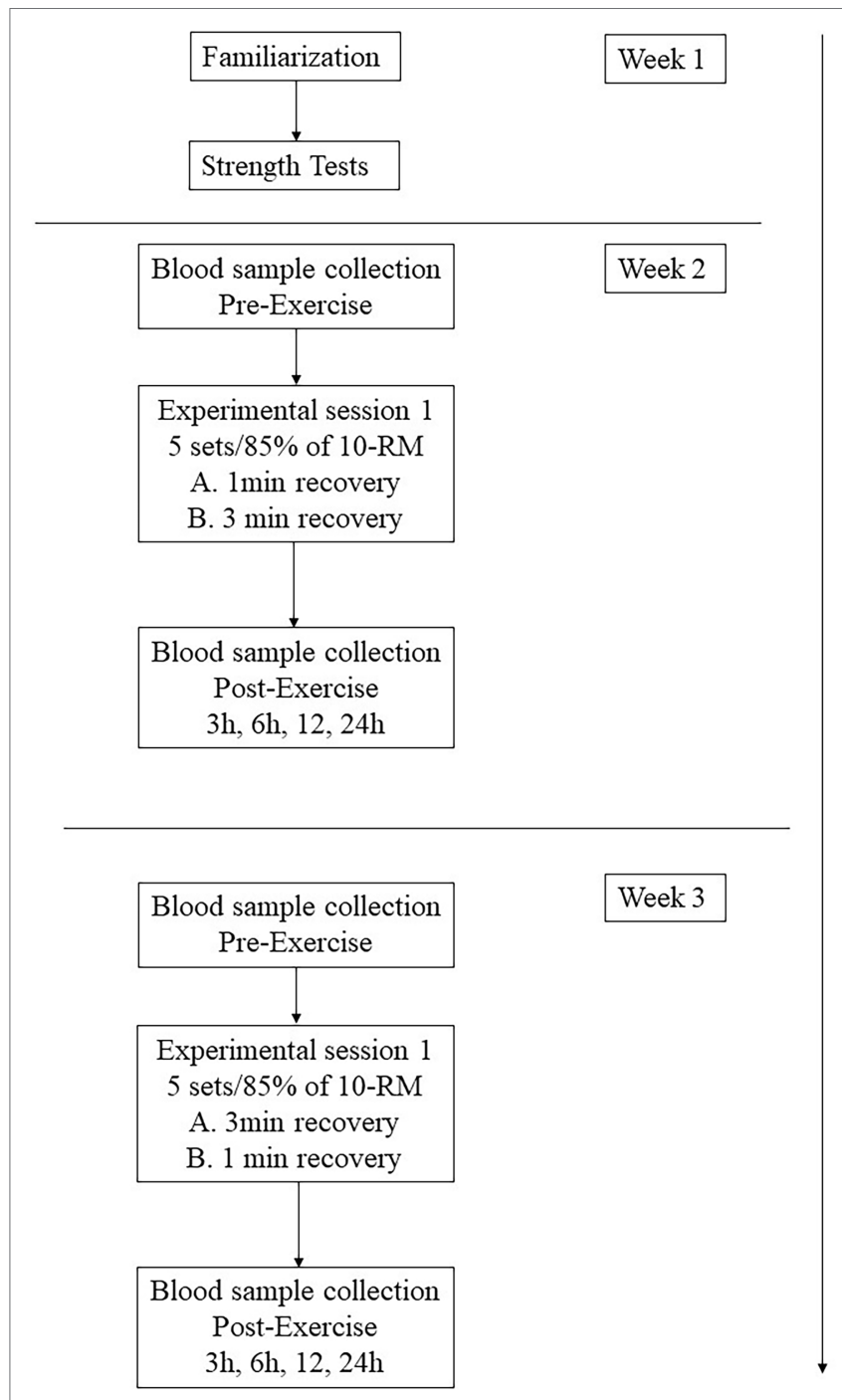

FIGURE 1 | Flowchart of the experimental approach to the problem.

sets) was counterbalanced and assigned through randomization. One week later, subjects completed the same workout protocol, with the remaining resting condition; in this manner, each participant performed both exercise protocols (Figure 1).

Before each workout, a standardized warm-up was performed consisting of two sets of 12 repetitions with $40 \%$ of a 10-RM load that was pre-estimated based on each subject's workout routine. A 2-min rest interval was observed after the warm-up and before the workout sets. No attempt was made to control the repetition velocity; however, subjects were required to use a smooth and controlled movement throughout range of motion (Senna et al., 2011). In order to avoid any potential confounding effects of the circadian cycle responses, all workout sessions were conducted between 06:00 a.m. and 08:00 a.m. Also, the time of data collection for a particular subject was held constant for each workout session (Scudese et al., 2016).

\section{Nutritional Control}

On the day before the first workout session, subjects recorded their dietary intake with an alimentary intake frequency questionnaire (AIFQ). After this verification, we observed that the participants ingested $2771.50 \pm 135.10 \mathrm{kcal}$ on the day before the first workout session, specifically: (a) carbohydrates: $1274.89 \pm 71.60 \mathrm{kcal}$; (b) protein: $914.60 \pm 44.58 \mathrm{kcal}$; and (c) fat: $582.02 \pm 28.37 \mathrm{kcal}$. Subjects received a copy of their diet record and were instructed to reproduce, as strictly as possible, this recorded dietary pattern on the day before the second workout. Subjects arrived at the laboratory after an overnight fast and were fed with a standard breakfast $1 \mathrm{~h}$ before each workout session in order to standardize the acute nutritional status. More specifically, the breakfast macronutrient distribution consisted of $40 \mathrm{~g}$ of carbohydrates, $8 \mathrm{~g}$ of protein, $6 \mathrm{~g}$ of fat, and $5 \mathrm{~g}$ of fiber, and totaling $256 \mathrm{kcal}$ of energy content.

The standardized meal was prepared to provide recommended amounts of macronutrient proportions according to the ACSM position stand on nutrition and athletic performance (Thomas et al., 2016). After the training sessions, subjects were oriented to maintain their regular nutritional habits via professional nutritionist guidance and were asked to avoid any extrapolation that might exacerbate the inflammatory response. Besides, in order to ensure that subjects arrived in a euhydrated state, they were instructed to ingest $5-7 \mathrm{ml}$ of water per kilogram of bodyweight immediately on awakening on workout days (Thomas et al., 2016).

\section{Statistical Analysis}

Variables are presented as means \pm SD. RPE is presented according to the median and interquartile range. A two-way ANOVA with repeated measures on both factors (rest condition vs. time points) was conducted for each blood analysis and ratio of the included variables. Fisher's least significant difference was used to identify pairwise differences when applicable. Effect sizes (ESs) for changes from Pre to 3, 6, 12, and $24 \mathrm{~h}$ were calculated. In order to interpret the magnitude of the ESs, the limits proposed by Cohen were adopted (Cohen, 1988). For blood concentrations data, the area under the curve (AUC) was calculated using the trapezoidal method and compared between rest conditions using a paired mean $t$ test. The Friedman test was used to investigate non-parametric data for RPE to compare differences in values between the distinct sets and rest protocols. When appropriate, Dunn's post hoc analysis was applied for multiple comparisons. Additionally, the Wilcoxon test was used for comparisons between RPE values resulting from different rest intervals. The level of significance assumed was $p \leq 0.05$. All statistics were performed via SPSS software, version 22.0 (IBM, Inc., United States).

\section{RESULTS}

\section{Muscle Tissue Damage Biomarkers}

A significant interaction (rest condition vs. time-point) for CK concentration (Figure 2) was observed $(p=0.02)$. Specifically, the main effect for time $(p<0.01)$, revealed increases after 6 , 

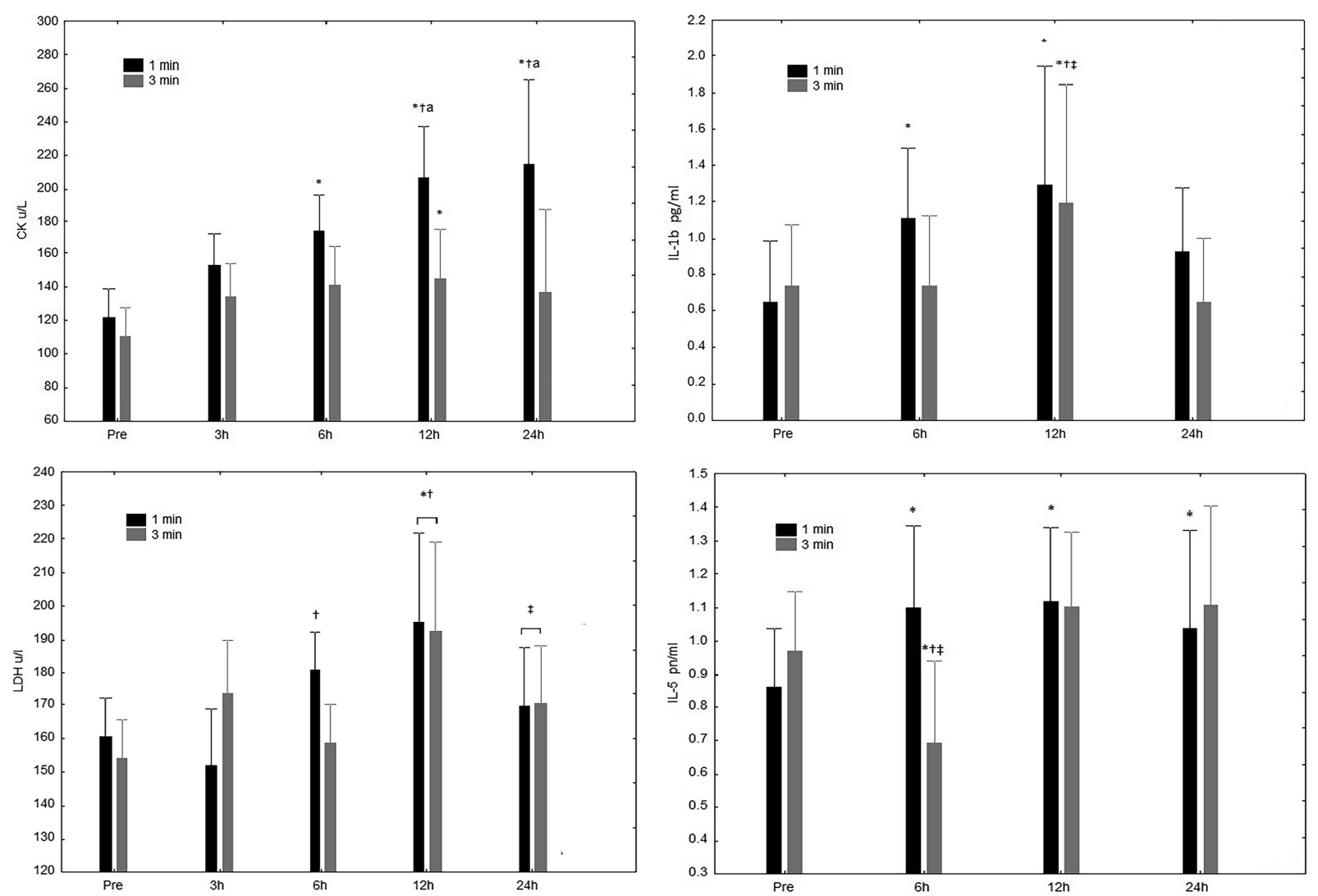

FIGURE 2 | CK/ul Creatine Kinase (CK) concentrations at pre-exercise (Pre), 3, 6, 12, and 24 h after completing five sets of bench press and leg press exercises with 1 or 3 min of rest between sets. LDH/ul L-Lactate Dehydrogenase (LDH) concentrations at pre-exercise (Pre), 3, 6, 12, and 24 h after completing five sets of bench press and leg press exercises with 1 or 3 min of rest between sets. *Significant difference to Pre ( $p \leq 0.05)$; ${ }^{+}$Significant difference to $3 \mathrm{~h}(p \leq 0.05)$; ${ }^{\circ}$ Significant

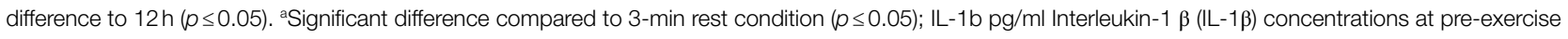
(Pre), 3, 6, 12, and 24h after completing five sets bench press and leg press exercises with 1 or 3 min of rest between sets. IL-5 pn/ml Interleukin-5 (IL-5) concentrations at pre-exercise (Pre), 3, 6, 12, and $24 \mathrm{~h}$ after completing five sets of bench press and leg press exercises with 1 or 3 min of rest between sets. *Significant difference to Pre $(p \leq 0.05)$; ${ }^{\dagger}$ Significant difference to $6 \mathrm{~h}(p \leq 0.05)$; ${ }^{*}$ Significant difference to $24 \mathrm{~h}(p \leq 0.05)$; and ${ }^{\text {}}$ significant difference compared to 3-min rest condition $(p \leq 0.05)$.

12 , and $24 \mathrm{~h}$ in the 1 -min rest condition; increases were observed only after $12 \mathrm{~h}$ in the 3 -min rest condition. Regarding the main effect between conditions ( $p=0.01)$ greater increases were observed after only after $12 \mathrm{~h}$. The AUC differed $(p<0.01)$ between the $1-\min \left(4572.4 \pm 1169.5 \mathrm{u} / \mathrm{L}_{\mathrm{h}} \mathrm{h}^{-1}\right)$ and $3-\min (3330.1 \pm 715.9 \mathrm{u} / \mathrm{L}$. $\mathrm{h}^{-1}$ ) condition (Figure 2). ESs appointed larges increases in $\mathrm{CK}$ magnitudes from 3 to $24 \mathrm{~h}$ time-points for 1 -min $(3 \mathrm{~h}$, $1.15 ; 6 \mathrm{~h}, 1.96 ; 12 \mathrm{~h}, 3.19$; and $24 \mathrm{~h}, 3.48)$ compared to the 3-min condition ( $3 \mathrm{~h}, 0.97$; $6 \mathrm{~h}, 1.04 \mathrm{12} \mathrm{h}, 1.07$; and $24 \mathrm{~h}, 1.00$ ).

No significant interaction for $\mathrm{LDH}$ concentration (Figure 2) was observed (rest condition vs. time-point). More specifically, both rest protocols resulted in significant increases in LDH after $12 \mathrm{~h}$; while a reduction was observed after $24 \mathrm{~h}$ regardless of the condition. For the main effect between conditions, no significant difference $(p=0.79)$ was observed.

\section{Leucocyte Count}

For the leucocyte count, we found a significant interaction (rest condition vs. time-point; $p<0.01$ ). For instance, the main effect regarding time $(p<0.01)$, revealed that for the 1 -min rest condition increases were present at 3,6 , and $12 \mathrm{~h}$; and significant reductions occurred between 12 and $24 \mathrm{~h}$ post exercise. For the main effect between rest conditions, no difference $(p=0.91)$ was found. The ESs presented larger increases in the total number of leukocytes for the 1-min rest condition. All values are reported in Table $\mathbf{1}$.

For neutrophils count, a significant interaction (rest condition vs. time-point; $p=0.03$ ) was observed. Significant increases were present at 3, 6, and $12 \mathrm{~h}$; while a reduction was present after $24 \mathrm{~h}$. No significant differences $(p=0.98)$ were found between conditions. All values are reported in Table $\mathbf{1}$.

No significant interaction (rest condition vs. time-point) was evinced in lymphocytes count $(p=0.09)$. All values are reported in Table $\mathbf{1}$.

A significant interaction (rest condition vs. time-point; $p<0.01$ ) for the monocytes count was found. More specifically, the for 1-min rest condition there was a significant increase after $12 \mathrm{~h}$ and a reduction after $24 \mathrm{~h}(p=0.03)$. The main effect 
TABLE 1 I Total number of leukocytes, neutrophils, lymphocytes, monocytes area under the curve (AUC), and effect size (ESs) at pre-exercise (Pre), $3 \mathrm{~h}$ ( $3 \mathrm{~h}), 6 \mathrm{~h}$ (6h), $12 \mathrm{~h}$ (12 h), and $24 \mathrm{~h}(24 \mathrm{~h})$ after completing five sets of bench press and leg press exercises with 1- or 3-min of rest between sets.

\begin{tabular}{|c|c|c|c|c|c|c|c|}
\hline $\begin{array}{l}\text { Rest } \\
\text { condition }\end{array}$ & Pre & $3 h$ & $6 \mathrm{~h}$ & $12 \mathrm{~h}$ & $24 h$ & AUC & $\begin{array}{c}\text { ESs (d) value } \\
\text { for AUC }\end{array}$ \\
\hline \multicolumn{8}{|c|}{ Total number of leucocytes $\left(\mathrm{mm}^{3}\right)$} \\
\hline $\begin{array}{l}1 \mathrm{~min} \\
\text { ESs (d) } \\
3 \mathrm{~min} \\
\text { ESs (d) }\end{array}$ & $\begin{array}{c}5901.9 \pm 966.9 \\
- \\
6555.9 \pm 1286.3 \\
-\end{array}$ & $\begin{array}{c}6664.1 \pm 1034.1 * \\
0.79 \\
6421.61 \pm 1256.2 \\
-0.10\end{array}$ & $\begin{array}{c}6816.9 \pm 1292.4 * \\
0.95 \\
6676.9 \pm 1,394,7 \\
0.09\end{array}$ & $\begin{array}{c}7879.5 \pm 915.3 * \#+ \\
2.05 \\
6951.8 \pm 1885.0 \\
0.30\end{array}$ & $\begin{array}{c}6432.4 \pm 1290.1^{\ddagger} \\
0.55 \\
6643.6 \pm 1087.9 \\
0.06\end{array}$ & $\begin{array}{l}169031.0 \pm 23175.4 \\
161574.0 \pm 33753.5\end{array}$ & 0.32 \\
\hline \multicolumn{8}{|c|}{ Neutrophils count $\left(\mathrm{mm}^{3}\right)$} \\
\hline $\begin{array}{l}1 \mathrm{~min} \\
\text { ESs (d) } \\
3 \mathrm{~min} \\
\text { ESs (d) }\end{array}$ & $\begin{array}{c}3230.7 \pm 794.6 \\
- \\
3645.7 \pm 1008.7 \\
-\end{array}$ & $\begin{array}{c}3980.3 \pm 876.8 * \\
0.94 \\
3799.5 \pm 1101.4 \\
0.85\end{array}$ & $\begin{array}{c}3912.6 \pm 909.7 * \\
0.86 \\
3807.1 \pm 1215.7 \\
0.86\end{array}$ & $\begin{array}{c}4361.0 \pm 1057.3^{* \dagger} \\
1.42 \\
3955.2 \pm 1124.8 \\
0.91\end{array}$ & $\begin{array}{c}3232.8 \pm 1008.5^{\#+\ddagger} \\
0.00 \\
3504.6 \pm 840.4 \\
0.74\end{array}$ & $\begin{array}{l}93039.5 \pm 20466.7 \\
90624.2 \pm 24203.5\end{array}$ & 0.12 \\
\hline \multicolumn{8}{|c|}{ Lymphocytes count $\left(\mathrm{mm}^{3}\right)$} \\
\hline $\begin{array}{l}1 \mathrm{~min} \\
\text { ESs (d) } \\
3 \text { min } \\
\text { ESs (d) }\end{array}$ & $\begin{array}{c}1885.6 \pm 470.5 \\
- \\
255.9 \pm 458.7 \\
-\end{array}$ & $\begin{array}{c}1827.2 \pm 473.9 \\
-0.12 \\
1883.3 \pm 360.6 \\
-0.38\end{array}$ & $\begin{array}{c}2126.1 \pm 193.5 \\
0.51 \\
2141.8 \pm 382.2^{\#} \\
0.19\end{array}$ & $\begin{array}{c}2583.1 \pm 444.9 * \#+ \\
1.48 \\
2158.9 \pm 765.5 \\
0.22\end{array}$ & $\begin{array}{c}2367.0 \pm 604.8^{* \#} \\
1.02 \\
2335.5 \pm 413.2^{\#} \\
0.61\end{array}$ & $\begin{array}{l}55328.4 \pm 8997.3 \\
51816.1 \pm 10326.1\end{array}$ & 0.390 \\
\hline \multicolumn{8}{|c|}{ Monocytes count $\left(\mathrm{mm}^{3}\right)$} \\
\hline $\begin{array}{l}1 \mathrm{~min} \\
\text { ESs (d) } \\
3 \mathrm{~min} \\
\text { ESs (d) }\end{array}$ & $\begin{array}{c}517.1 \pm 133.8 \\
- \\
597.7 \pm 75.2 \\
-\end{array}$ & $\begin{array}{c}528.5 \pm 80.2 \\
0.09 \\
566.8 \pm 89.1 \\
-0.41\end{array}$ & $\begin{array}{c}543.2 \pm 75.3 \\
0.21 \\
574.2 \pm 89.9 \\
-0.31\end{array}$ & $\begin{array}{c}639.1 \pm 94.2^{* \#+} \\
0.91 \\
571.3 \pm 63.7 \\
-0.35\end{array}$ & $\begin{array}{c}542.5 \pm 133.2^{\ddagger} \\
0.19 \\
579.7 \pm 96.6 \\
-0.24\end{array}$ & $\begin{array}{l}13812.3 \pm 2113.3 \\
13801.7 \pm 1594.8\end{array}$ & 0.005 \\
\hline
\end{tabular}

* Significant difference to Pre $(p \leq 0.05)$

"Significant difference to $3 h(p \leq 0.05)$;

+Significant difference to $6 h(p \leq 0.05)$;

¥Significant difference to $12 \mathrm{~h}(\mathrm{p} \leq 0.05)$.

between conditions however did not show any significant difference $(p=0.50)$. All values are reported in Table 1 .

\section{Cytokines Concentrations}

A significant interaction was observed for IL-1 $\beta$ concentration for time (Figure 2; but not rest condition vs. time-point; $p=0.43$ ). The 1 -min rest protocol resulted in significant increases in Il-1 $\beta$ after 6 and $12 \mathrm{~h}$; the 3-min rest interval showed an increase only after $12 \mathrm{~h}$. No significant interaction was found between conditions.

For IL-5 concentration (Figure 2), a significant interaction effect was observed (rest condition vs. time point; $p<0.01$ ). Significant increases in IL-5 were observed after 6,12 , and $24 \mathrm{~h}$ in the 1-min condition; decreased values were observed after $6 \mathrm{~h}$ for the 3 -min rest interval. No significant interaction $(p=0.65)$ was seen between conditions.

For IL-6 concentration (Figure 3), a significant interaction was observed (rest condition vs. time point; $p=0.04$ ). Significant increases in IL-6 were present after 6,12 , and $24 \mathrm{~h}$ in the 1 -min condition, while only after $12 \mathrm{~h}$ increases were observed in the 3-min rest interval. No significant interaction $(p=0.65)$ was seen between conditions.

Differences in IL-10 concentration (Figure 3), were not observed (rest condition vs. time point; $p=0.27$ ). However, significant increases in Il-10 after 6,12 , and $24 \mathrm{~h}$ were present following the 1-min condition; and for the 3-min rest interval only after $12 \mathrm{~h}$ compared to all other time-points. No significant interaction $(p=0.65)$ was observed between conditions.
For GM-CSF concentration (Figure 3), a significant interaction (rest condition vs. time point) was observed $(p=0.04)$. More specifically, a main effect for time $(p<0.01)$, was observed after 6,12 , and $24 \mathrm{~h}$ in the 1 -min condition, while an increase was present only after $12 \mathrm{~h}$ following the 3 -min rest interval.

For TNF- $\alpha$ concentration (Figure 3), there were no significant interactions (rest condition vs. time point; $p=0.13$ ). However, regarding time in the 1-min rest increases were observed after 12 and $24 \mathrm{~h}$; while differences were present after the 3-min condition only after $12 \mathrm{~h}$. No significant difference was present between rest conditions $(p=0.10)$.

For IL-10/TNF- $\alpha$ concentration, there was no significant interaction (rest condition vs. time point; $p=0.84$ ). More specifically, no difference neither for time $(p=0.21)$ nor condition $(p=0.40)$ were observed (Figure 4). However, the AUC did differ $(p=0.03)$ between the $1-\min \left(41.50 \pm 9.18 \mathrm{pn} / \mathrm{ml}^{-1} \mathrm{~h}^{-1}\right)$ and 3 - $\mathrm{min}\left(26.98 \pm 10.70 \mathrm{pn} / \mathrm{ml}^{-\mathrm{h}^{-1}}\right)$ rest conditions.

\section{Rate of Perceived Exertion}

Significantly higher values of post-set RPE were evident from the third set for the shorter 1-min rest condition compared to the first set, and only from the fourth set for the more extended 3-min rest condition compared to the first set. Additionally, significant differences were also observed in post-set RPE values, from the second set between conditions (SH, $p<0.01$; LP, $p<0.01)$. All RPE data are presented in Table 2. 

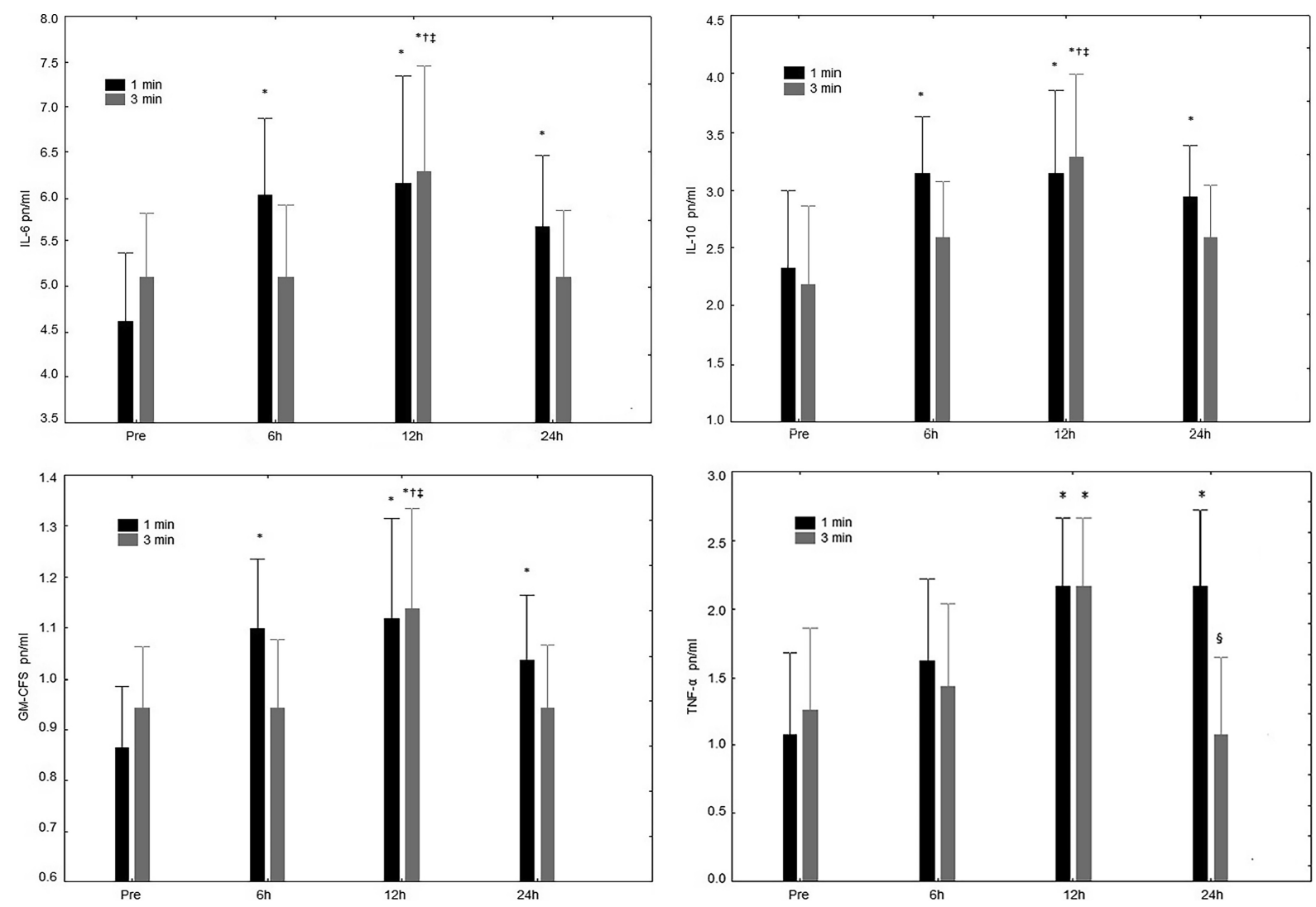

FIGURE 3 | IL-5 pn/ml Interleukin-6 (IL-6) concentrations at pre-exercise (Pre), 3, 6, 12, and 24 h after completing five sets of bench press and leg press exercises with 1 or $3 \mathrm{~min}$ of rest between sets. IL-10 pn/ml Interleukin-10 (IL-10) concentrations at pre-exercise (Pre), 3, 6, 12, and 24 h after completing five sets of bench press and leg press exercises with 1 or 3 min of rest between sets. GM-CFS pn/ml Granulocyte-macrophage colonystimulating factor (GM-CSF) concentrations at pre-exercise (Pre), 3, 6, 12, and $24 \mathrm{~h}$ after completing five sets of bench press and leg press exercises with 1- or 3-min of rest between sets. $\mathrm{TNF}-\alpha \mathrm{pn} / \mathrm{ml}$ Tumoral necrosis factor (TNF- $\alpha$ ) concentrations at pre-exercise (Pre), 3, 6, 12, and $24 \mathrm{~h}$ after completing 5 sets of bench press and leg press exercises with 1 - or 3 -min of rest between sets. *Significant difference to Pre $(p \leq 0.05)$; ${ }^{\dagger}$ Significant difference to 6 h $(p \leq 0.05)$; ${ }^{\S}$ Significant difference to $12 \mathrm{~h}(p$ $\leq 0.05)$; and $¥$ Significant difference to $24 \mathrm{~h}(p \leq 0.05)$.

\section{DISCUSSION}

Our key findings regard the muscle tissue damage (CK) triggered by the shorter inter-set length (1-min) observed from the early $12 \mathrm{~h}$ to the $24 \mathrm{~h}$ post-exercise window, compared to the 3 -min rest condition. Increased muscle damage contributed to the increases observed in the total number of leukocytes, neutrophils (3-12 $\mathrm{h}$ post-exercise), and monocytes ( $12 \mathrm{~h}$ post-exercise) in the shorter inter-set rest condition. Altogether, increased inflammatory process due to increased muscle damage, increased pro-inflammatory cytokines (IL- $1 \beta, \mathrm{TNF}-\alpha$, and GM-CSF) mainly after 6 and $12 \mathrm{~h}$. Following the aforementioned increase, an increase in anti-inflammatory cytokines (IL-5, IL-6, and IL-10) was observed $(6,12$, and $24 \mathrm{~h})$. In accordance with these findings, the shorter rest period length triggered significantly higher RPE values compared to the longer rest condition for both volume-equated training protocols.

The level of serum $\mathrm{CK}$ and $\mathrm{LDH}$ can be elevated due to muscle tissue damage as a consequence of high-intensity training (Rodrigues et al., 2010). These increases may be a response to metabolic and mechanical stimuli (Brancaccio et al., 2007). One of the mechanisms may be related to local tissue damage with degeneration and sarcomeric fragmentation between the $Z$ discs (Brancaccio et al., 2007). Another mechanism that seems to be associated with this damage is metabolic exhaustion of muscle fibers, which in turn presents a decrease in membrane resistance after an elevation in free internal calcium ions, as well as the activation of the potassium channels, occurred by the reductions in cellular energy reserves (Fink and Luttgau, 1976). Therefore, the response pattern of these enzymes (CK and $\mathrm{LDH}$ ) suggests an appropriate indicator of the intensity of a previously performed exercise (Bessa et al., 2016). Therefore, it is plausible to interpret that different manipulations in $\mathrm{RE}$ variables can trigger distinct biological responses (Helms et al., 2020). Nevertheless, other studies have indeed focused on the impact of rest interval manipulation on muscle tissue damage and immune responses (Rodrigues et al., 2010; Evangelista et al., 2011; Machado et al., 2012; Gerosa-Neto et al., 2016; Rossi et al., 2016). However, these present great variations in blood time-point choices, populations, non-equalized vs. 


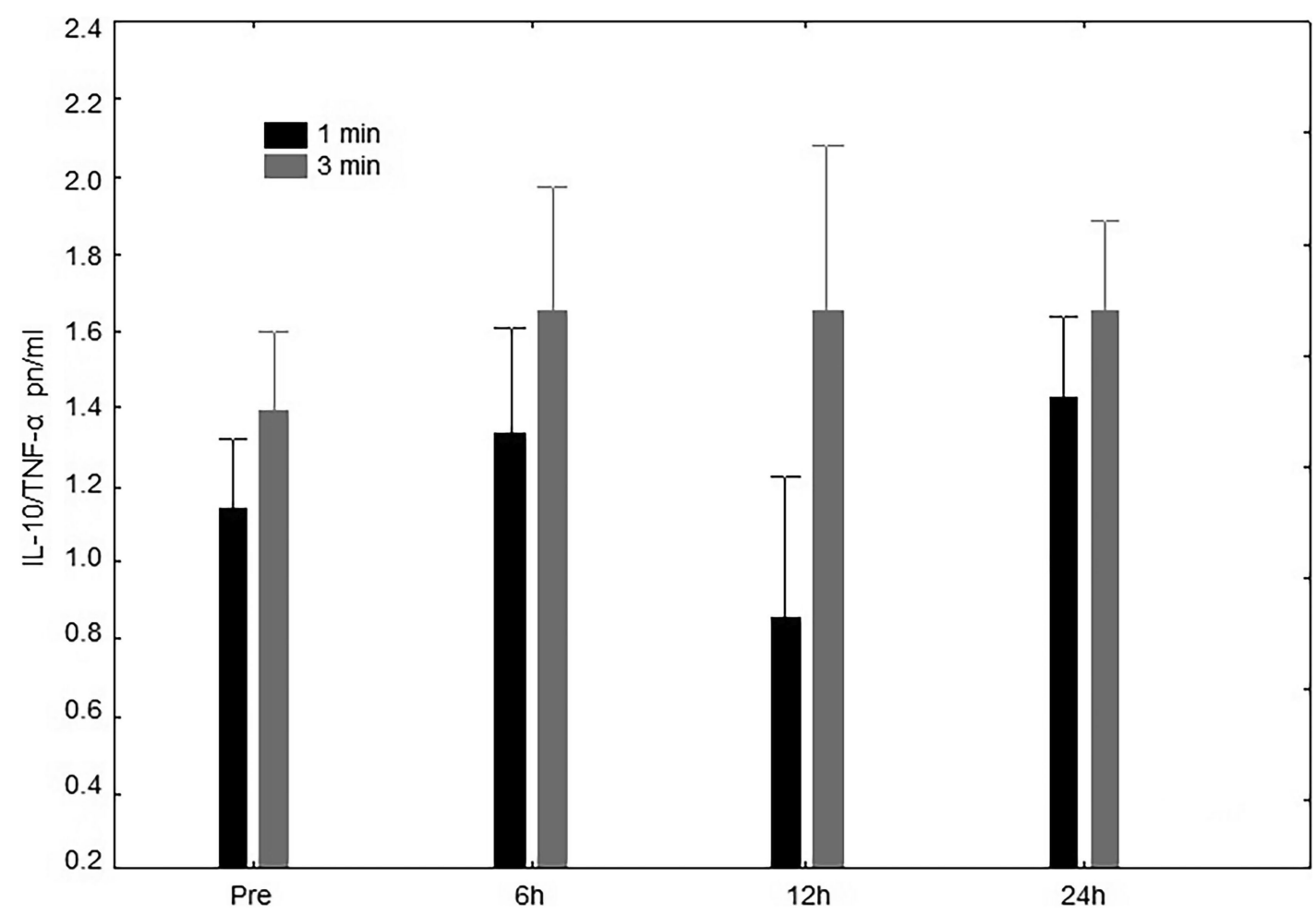

FIGURE 4 | IL-10/TNF- $\alpha$ pn/ml Interleukin-10 and tumoral necrosis factor- $\alpha$ ratio (IL-10/TNF- $\alpha$ ) concentrations at pre-exercise (Pre), 3,6 , 12 , and 24 h after completing five sets of bench press and leg press exercises with 1- or 3-min of rest between sets.

TABLE 2 | Rating of perceived exertion (RPE). Post-set values for both exercises for the 1- and 3-min rest conditions [median (25\%-75\%)].

\begin{tabular}{|c|c|c|c|c|c|}
\hline & Set 1 & Set 2 & Set 3 & Set 4 & Set 5 \\
\hline \multicolumn{6}{|c|}{ Bench press } \\
\hline 1-min & $4(3-4)$ & $5(5-5.75)$ & $7(6.25-7.5)^{*}$ & $8(8-8.5)^{* \#}$ & $9(9-9.5)^{* \#}$ \\
\hline 3-min & $4(3.25-4)$ & $4.25(4-4.5)^{a}$ & $4.75(4.5-5)^{a}$ & $6(5-6.5)^{* a}$ & $7(5-7)^{* \# a}$ \\
\hline \multicolumn{6}{|c|}{ Leg press } \\
\hline 1-min & $7(7-7.5)$ & $8(8-8)$ & $9(8-9) *$ & 9 (9-9)* & $10(9.5-10) * \#$ \\
\hline 3-min & $6.25(5-7)$ & $7(5.5-7.5)^{\mathrm{a}}$ & $7(6-8)^{a}$ & $7.5(6.5-8.5)^{* \# a}$ & $7.5(7-8.5)^{* \# a}$ \\
\hline
\end{tabular}

* Significant difference compared to Set 1;

"Significant difference compared to Set 2;

aSignificant difference compared to 1-min rest condition.

equalized volume and exercise schemes, relative loads, and nutritional controls. All this variability, unfortunately, prevents a consensus regarding post-exercise muscle tissue damage and immune responses patterns in acute response to distinct inter-set lengths.

Rodrigues et al. (2010) compared CK and LDH concentrations at multiple post-exercise time-points $(>24 \mathrm{~h})$ after RE sessions with different inter-set intervals. The two experimental sessions consisted of three sets with loads of $80 \%$ of $1-\mathrm{RM}$ until concentric failure, which caused a $24 \%$ larger volume for the longer interval (non-equalized method). There were no significant differences in $\mathrm{CK}$ and $\mathrm{LDH}$ concentrations at any post-exercise assessment between the different intervals. Other authors observed the effects of different rest intervals on CK and LDH in sessions with 10-RM loads until concentric failure (Machado et al., 2011). The intervals stipulated in this experiment were 1-, 1.5-, 2-, and 3-min between sets and exercises. The $\mathrm{CK}$ and $\mathrm{LDH}$ were significantly elevated after 24 and $72 \mathrm{~h}$ in all sessions and without differences between the distinct intervals. When comparing these studies (Rodrigues et al., 2010; Machado et al., 2011) with the present experiment, there is an evident methodological difference, mainly at the proposed attempt to analyze the interval time between sets without any control of other variables as volume or intensity.

It is important to underline, that in practical terms different rest periods are chosen to allow full (longer interest periods) or incomplete recovery (shorter interest periods) between sets. In the case of longer rest periods, this avoids muscle impairments, with the consequence to counteract load decreases caused by fatigue (Schoenfeld et al., 2016), which may occur if using interest length shorter than 2 min (Matos et al., 2021). Despite perceived exertion may result increased with lower-rest intervals (Scudese et al., 2015), a progressive load decrease along with subsequent sets determines a decrease in both total volume and intensity (Matos et al., 2021), which could result in lower gains in terms of hypertrophy and strength (Schoenfeld et al., 2015, 2019).

In another investigation (Evangelista et al., 2011) which compared the differences between two distinct rest interval lengths (1-vs. 3-min) on volume, muscle damage, and muscle 
soreness, the subjects performed an experimental protocol consisting of three sets of biceps exercise with $40 \%$ of maximal voluntary contraction. The results showed that individuals who performed the exercises with the longer rest intervals performed more volume (as expected). Still, there were no differences for CK ( 24 and $48 \mathrm{~h}$ after exercise) and muscle soreness between groups, probably due to the low intensity performed. Similarly, Machado et al. (2012) examined the values of CK activity after RE sessions in subjects that the authors classified as possessing high, medium, or low responsiveness to the exercises. Individuals classified with high and medium responsiveness demonstrated an elevated CK activity after the 1-min interval session compared to the 3-min interval session. However, it is important to note that the blood examinations were restricted to 24 and $48 \mathrm{~h}$ post-exercise, and the training routine consisted of only one single-joint exercise (bicep curl).

By analyzing the previous studies' methodology, it is safe to state that our experiment is original regarding the training volume equalization and the meticulously tailored timing of $\mathrm{CK}$ and LDH blood examinations. More specifically, our results allowed to observe that the shorter inter-set period potentiated the muscle damage markers responses. It should also be noted that the main focus of the present study was the initial and rapidly (up to $24 \mathrm{~h}$ ) curve identification of $\mathrm{CK}$ and $\mathrm{LDH}$ responses, demonstrating an early post-exercise sensibility of those biomarkers. Moreover, the CK increase had its onset as early as $6 \mathrm{~h}$ post-exercise for the 1-min interval, and the LDH showed its peak around the $12 \mathrm{~h}$ post-exercise assessment. These data bring to light new evidence that the majority of the previous investigations might have missed the sensitivity window for those responses.

Regarding the pro- and anti-inflammatory process resulting from the RE responses, shortly after the muscle damage occurrence, there was leukocyte mobilization followed by its migration to the injured muscle tissue (Peake et al., 2006). The neutrophils and monocytes, the cellular types mobilized in greater magnitude at different moments in the process of muscle tissue remodeling, are responsible for the reduction of the damaged muscular tissue and the release of pro- and antiinflammatory cytokines.

Several studies on the inflammatory responses to RE (Peake et al., 2006; Phillips et al., 2010) showed that the total training volume (rather than intensity) directly influences pro-inflammatory interleukins up to $12 \mathrm{~h}$ after training. Smith et al. (2000) and Hirose et al. (2004) observed important modifications for pro-inflammatory interleukins in assessments performed up to $72 \mathrm{~h}$ after exercise even with low training volumes. Despite evidence exists regarding manipulation of $\mathrm{RE}$ volume, little is known regarding inter-set differences and inflammatory responses. An investigation by Kraemer et al. (1996) in 1996 examined the impact of exercise-induced circulating plasma cortisol elevations and leukocyte counts with different inter-set lengths. Venous blood samples were obtained pre, during exercise, and 5-min after exercise. There were no significant variations in leukocyte differential counts. Probably, due to the very close assessment point, $5 \mathrm{~min}$ post-exercise. Though, seeking to determine the effect of distinct rest intervals on leukocyte levels during moderateintensity RE, Mayhew et al. (2005) performed a study where nine men completed exercise sessions with 1 vs. $3 \mathrm{~min}$ inter-set intervals. Blood was collected at rest, immediately after, 60-, and 90-min post-exercise, and the leukocyte concentration was analyzed. Increased lymphocytosis and monocytosis were observed after the 1-min inter-set length protocol but not after the 3-min inter-set length protocol. Although the studies by Kraemer et al. (1996) and the more recent of Mayhew et al. (2005) have verified different inter-set length and their responses to leukocyte count, important limitations, such as the timing of the blood sample collection may have compromised the outcomes retrieved. In the present study, the leukocyte count was aligned with the CK and $\mathrm{LDH}$ assessments during the process of tissue damage (up to $24 \mathrm{~h}$ after exercise). In such consideration, possibly after muscle damage in response to high-intensity RE, occurred an important leukocyte mobilization and invasion (mainly neutrophils) to the injured tissue alongside with a significant monocyte elevation.

More recently, a two-part experiment (Gerosa-Neto et al., 2016; Rossi et al., 2016) aimed to verify the influence of a very short (30-s) and moderate (90-s) inter-set length on performance, inflammatory, and metabolic responses in healthy adults and recreational weightlifters. In part 1, eight healthy subjects performed two exercises at $70 \%$ of 1 -RM; and in part 2, the procedures were repeated; however, with $90 \%$ of 1-RM. Both conditions each with the different inter-set lengths. The TNF- $\alpha$, IL- 6 , and IL-10 were assessed at baseline, immediately after exercise, 15 and $30 \mathrm{~min}$ postexercise. The authors concluded that exhaustive and heavy strength exercises conducted with different inter-set length decreased performance; however, an augmented inflammatory and metabolic response were found only in the longer 90-s interval. Despite similarities in inter-set length are observed when comparing these studies to our experiment, differences occur in the timing of the post-exercise blood assessments and the volume equalization.

Other finding of this investigation, is that related to perceived exertion of the participants. We demonstrated significant increases in RPE with the progression of the sets for both exercises, with the highest values found for the shorter 1-min inter-set length. To date, only two investigations compared different rest conditions on the RPE responses in submaximal exercises, both observing that the shorter rests, increased RPE responses (Scudese et al., 2015, 2016). Other experiments focused on the RPE influences of different rest intervals between sets in distinct types of exercise (Senna et al., 2011) and near-maximum loads zones (Scudese et al., 2015; Senna et al., 2016).

Limitations to our study are the relatively small sample size, since not many participants who met all the required inclusion criteria were willing to participate due to the repeated blood sample collection. Further, our evaluation up to $24 \mathrm{~h}$ post-exercise only is a further limitation as muscle damage and inflammation typically occur up until $48 \mathrm{~h}$ post-exercise. However, it was aim of this study to evaluate the curve response within the first $24 \mathrm{~h}$. The inclusion of a third 5-min inter-rest condition could have 
provided further confirmation regarding the data included for the 3-min inter-rest length. Further consideration could be that the intensity used across the two protocols was $85 \%$ of $10 \mathrm{RM}$, which may not be of sufficient intensity to produced muscle damage with long rest periods and may have produced different responses compared to repetition maximum training.

\section{PRACTICAL APPLICATIONS}

This study brings a new approach to the way muscle damage and inflammatory response are investigated after a resistance training routine. Our results suggest that when equalized for training volume, a shorter inter-set length promotes a considerably greater damage to muscle tissues, as well as a longer duration of the inflammatory process of this tissue. Based on these findings, specifically on the durable inflammatory augmentation observed with the shorter inter-set length, practitioners with the intent to promote hypertrophy or local muscle endurance development should consider implementing shorter inter-set lengths when using a similar type of training method. Other important aspect to consider is that shorter inter-set periods allow shorter workouts, which in turn improve training efficiency.

This data might contribute to future recommendations focused on different goals requiring considerable muscle tissue damage and inflammatory response for its optimization. These results are applicable and limited to the specific exercises, inter-set length, and load examined. However, we strongly recommend that future studies should evaluate distinct exercise schemes,

\section{REFERENCES}

American College of Sports Medicine (2009). American College of Sports Medicine position stand. Progression models in resistance training for healthy adults. Med. Sci. Sports Exerc. 41, 687-708. doi: 10.1249/MSS.0b013e3181915670

Baechle, T.R., and Earle, R.W. (2000). Essentials of Strength Training and Conditioning. 2nd Edn. Illinois, US: Human Kinetics.

Bessa, A. L., Oliveira, V. N., Agostini, G. G., Oliveira, R. J., Oliveira, A. C., White, G. E., et al. (2016). Exercise intensity and recovery: biomarkers of injury, inflammation, and oxidative stress. J. Strength Cond. Res. 30, 311-319. doi: 10.1519/JSC.0b013e31828f1ee9

Borde, R., Hortobágyi, T., and Granacher, U. (2015). Dose-response relationships of resistance training in healthy old adults: a systematic review and metaanalysis. Sports Med. 45, 1693-1720. doi: 10.1007/s40279-015-0385-9

Brancaccio, P., Maffulli, N., and Limongelli, F. M. (2007). Creatine kinase monitoring in sport medicine. Br. Med. Bull. 81-82, 209-230. doi: 10.1093/bmb/ldm014

Calle, M. C., and Fernandez, M. L. (2010). Effects of resistance training on the inflammatory response. Nutr. Res. Pract. 4, 259-269. doi: 10.4162/nrp.2010.4.4.259

Chatzinikolaou, A., Fatouros, I. G., Gourgoulis, V., Avloniti, A., Jamurtas, A. Z., Nikolaidis, M. G., et al. (2010). Time course of changes in performance and inflammatory responses after acute plyometric exercise. J. Strength Cond. Res. 24, 1389-1398. doi: 10.1519/JSC.0b013e3181d1d318

Cohen, J. (1988). Statistical Power Analysis for the Behavioral Sciences. 2nd Edn. Hillsdale, NJ: Lawrence Erlbaum.

de Salles, B. F., Simao, R., Miranda, F., Novaes Jda, S., Lemos, A., and Willardson, J. M. (2009). Rest interval between sets in strength training. Sports Med. 39, 765-777. doi: 10.2165/11315230-000000000-00000 other load ranges, types of rest period (active vs. passive), and whole-body training sessions.

\section{DATA AVAILABILITY STATEMENT}

The raw data supporting the conclusions of this article will be made available by the authors, after reasonable request.

\section{ETHICS STATEMENT}

The studies involving human participants were reviewed and approved by the Federal University of State of Rio de Janeiro ethics committee (CAAE 63803717.2.0000.5285). The patients/ participants provided their written informed consent to participate in this study.

\section{AUTHOR CONTRIBUTIONS}

All authors listed have made a substantial, direct, and intellectual contribution to the work and approved it for publication.

\section{FUNDING}

This study was financed in part by the Coordenação de Aperfeiçoamento de Pessoal de Nível Superior - Brasil (CAPES)Finance Code 001.
Evangelista, R., Pereira, R., Hackney, A. C., and Machado, M. (2011). Rest interval between resistance exercise sets: length affects volume but not creatine kinase activity or muscle soreness. Int. J. Sports Physiol. Perform. 6, 118-127. doi: 10.1123/ijspp.6.1.118

Fatouros, I. G., and Jamurtas, A. Z. (2016). Insights into the molecular etiology of exercise-induced inflammation: opportunities for optimizing performance. J. Inflamm. Res. 9, 175-186. doi: 10.2147/jir.s114635

Fink, R., and Luttgau, H. C. (1976). An evaluation of the membrane constants and the potassium conductance in metabolically exhausted muscle fibres. J. Physiol. 263, 215-238. doi: 10.1113/jphysiol.1976.sp011629

Fukada, S. I., Akimoto, T., and Sotiropoulos, A. (2020). Role of damage and management in muscle hypertrophy: different behaviors of muscle stem cells in regeneration and hypertrophy. Biochim. Biophys. Acta, Mol. Cell Res. 1867:118742. doi: 10.1016/j.bbamcr.2020.118742

Garber, C. E., Blissmer, B., Deschenes, M. R., Franklin, B. A., Lamonte, M. J., Lee, I. M., et al. (2011). American College of Sports Medicine position stand. Quantity and quality of exercise for developing and maintaining cardiorespiratory, musculoskeletal, and neuromotor fitness in apparently healthy adults: guidance for prescribing exercise. Med. Sci. Sports Exerc. 43, 1334-1359. doi: 10.1249/MSS.0b013e318213fefb

Gerosa-Neto, J., Monteiro, P. A., Inoue, D. S., Antunes, B. M., Batatinha, H., Dorneles, G. P., et al. (2020). High- and moderate-intensity training modify LPS-induced ex-vivo interleukin-10 production in obese men in response to an acute exercise bout. Cytokine 136:155249. doi: 10.1016/j. cyto. 2020.155249

Gerosa-Neto, J., Rossi, F. E., Campos, E. Z., Antunes, B. M., Cholewa, J. M., Lira, F. S., et al. (2016). Impact of short and moderate rest intervals on the acute Immunometabolic response to exhaustive strength exercise: 
part II. J. Strength Cond. Res. 30, 1570-1576. doi: 10.1519/ JSC. 0000000000001413

Grgic, J., Lazinica, B., Mikulic, P., Krieger, J. W., and Schoenfeld, B. J. (2017). The effects of short versus long inter-set rest intervals in resistance training on measures of muscle hypertrophy: A systematic review. Eur. J. Sport Sci. 17, 983-993. doi: 10.1080/17461391.2017.1340524

Helms, E. R., Kwan, K., Sousa, C. A., Cronin, J. B., Storey, A. G., and Zourdos, M. C. (2020). Methods for regulating and monitoring resistance training. J. Hum. Kinet. 74, 23-42. doi: 10.2478/hukin-2020-0011

Henselmans, M., and Schoenfeld, B. J. (2014). The effect of inter-set rest intervals on resistance exercise-induced muscle hypertrophy. Sports Med. 44, 1635-1643. doi: $10.1007 /$ s40279-014-0228-0

Hirose, L., Nosaka, K., Newton, M., Laveder, A., Kano, M., Peake, J., et al. (2004). Changes in inflammatory mediators following eccentric exercise of the elbow flexors. Exerc. Immunol. Rev. 10, 75-90.

Kilgore, J. L., Pendlay, G. W., Reeves, J. S., and Kilgore, T. G. (2002). Serum chemistry and hematological adaptations to 6 weeks of moderate to intense resistance training. J. Strength Cond. Res. 16, 509-515.

Kraemer, W. J., Clemson, A., Triplett, N. T., Bush, J. A., Newton, R. U., and Lynch, J. M. (1996). The effects of plasma cortisol elevation on total and differential leukocyte counts in response to heavy-resistance exercise. Eur. J. Appl. Physiol. Occup. Physiol. 73, 93-97. doi: 10.1007/ BF00262815

Lagally, K. M., and Robertson, R. J. (2006). Construct validity of the OMNI resistance exercise scale. J. Strength Cond. Res. 20, 252-256. doi: 10.1519/R17224.1

Machado, M., Koch, A. J., Willardson, J. M., Pereira, L. S., Cardoso, M. I., Motta, M. K. S., et al. (2011). Effect of varying rest intervals between sets of assistance exercises on creatine kinase and lactate dehydrogenase responses. J. Strength Cond. Res. 25, 1339-1345. doi: 10.1519/ JSC.0b013e3181d680d6

Machado, M., Pereira, R., and Willardson, J. M. (2012). Short intervals between sets and individuality of muscle damage response. J. Strength Cond. Res. 26, 2946-2952. doi: 10.1519/JSC.0b013e318243fdb5

Machado, M., and Willardson, J. M. (2010). Short recovery augments magnitude of muscle damage in high responders. Med. Sci. Sports Exerc. 42, 1370-1374. doi: 10.1249/MSS.0b013e3181ca7e16

Margaritelis, N. V., Theodorou, A. A., Chatzinikolaou, P. N., Kyparos, A., Nikolaidis, M. G., and Paschalis, V. (2021). Eccentric exercise per se does not affect muscle damage biomarkers: early and late phase adaptations. Eur. J. Appl. Physiol. 121, 549-559. doi: 10.1007/s00421-020-04528-w

Markus, I., Constantini, K., Hoffman, J. R., Bartolomei, S., and Gepner, Y. (2021). Exercise-induced muscle damage: mechanism, assessment and nutritional factors to accelerate recovery. Eur. J. Appl. Physiol. 121, 969-992. doi: $10.1007 / \mathrm{s} 00421-020-04566-4$

Matos, F., Ferreira, B., Guedes, J., Saavedra, F., Reis, V. M., and Vilaça-Alves, J. (2021). Effect of rest interval between sets in the muscle function During a sequence of strength training exercises for the upper body. J. Strength Cond. Res. 1628-1635. doi: 10.1519/jsc.0000000000002941 [Epub ahead of print].

Mayhew, D. L., Thyfault, J. P., and Koch, A. J. (2005). Rest-interval length affects leukocyte levels during heavy resistance exercise. J. Strength Cond. Res. 19, 16-22. doi: 10.1519/R-14113.1

Minari, A. L. A., and Thomatieli-Santos, R. V. (2021). From skeletal muscle damage and regeneration to the hypertrophy induced by exercise: what is the role of different macrophages subsets? Am. J. Phys. Regul. Integr. Comp. Phys. 322, R41-R54. doi: 10.1152/ajpregu.00038.2021

Peake, J. M., Nosaka, K., Muthalib, M., and Suzuki, K. (2006). Systemic inflammatory responses to maximal versus submaximal lengthening contractions of the elbow flexors. Exerc. Immunol. Rev. 12, 72-85.

Pedersen, B. K., and Hoffman-Goetz, L. (2000). Exercise and the immune system: regulation, integration, and adaptation. Physiol. Rev. 80, 1055-1081. doi: 10.1152/physrev.2000.80.3.1055

Phillips, M. D., Mitchell, J. B., Currie-Elolf, L. M., Yellott, R. C., and Hubing, K. A. (2010). Influence of commonly employed resistance exercise protocols on circulating IL-6 and indices of insulin sensitivity. J. Strength Cond. Res. 24, 1091-1101. doi: 10.1519/JSC.0b013e3181cc2212

Raastad, T., Risoy, B. A., Benestad, H. B., Fjeld, J. G., and Hallen, J. (1985). Temporal relation between leukocyte accumulation in muscles and halted recovery 10-20 h after strength exercise. J. Appl. Physiol. 95, 2503-2509. doi: $10.1152 /$ japplphysiol.01064.2002

Ratamess, N. A., Falvo, M. J., Mangine, G. T., Hoffman, J. R., Faigenbaum, A. D., and Kang, J. (2007). The effect of rest interval length on metabolic responses to the bench press exercise. Eur. J. Appl. Physiol. 100, 1-17. doi: 10.1007/s00421-007-0394-y

Rodrigues, B. M., Dantas, E., de Salles, B. F., Miranda, H., Koch, A. J., Willardson, J. M., et al. (2010). Creatine kinase and lactate dehydrogenase responses after upperbody resistance exercise with different rest intervals. J. Strength Cond. Res. 24, 1657-1662. doi: 10.1519/JSC.0b013e3181d8e6bl

Rossi, F. E., Gerosa-Neto, J., Zanchi, N. E., Cholewa, J. M., and Lira, F. S. (2016). Impact of short and moderate rest intervals on the acute immunometabolic response to exhaustive strength exercise: part I. J. Strength Cond. Res. 30, 1563-1569. doi: 10.1519/JSC.0000000000001189

Schoenfeld, B. J. (2012). Does exercise-induced muscle damage play a role in skeletal muscle hypertrophy? J. Strength Cond. Res. 26, 1441-1453. doi: 10.1519/JSC.0b013e31824f207e

Schoenfeld, B. J., Contreras, B., Krieger, J., Grgic, J., Delcastillo, K., Belliard, R., et al. (2019). Resistance training volume enhances muscle hypertrophy but not strength in trained men. Med. Sci. Sports Exerc. 51, 94-103. doi: 10.1249/ MSS.0000000000001764

Schoenfeld, B. J., Peterson, M. D., Ogborn, D., Contreras, B., and Sonmez, G. T. (2015). Effects of low- vs. high-load resistance training on muscle strength and hypertrophy in well-trained men. J. Strength Cond. Res. 29, 2954-2963. doi: 10.1519/JSC.0000000000000958

Schoenfeld, B. J., Pope, Z. K., Benik, F. M., Hester, G. M., Sellers, J., Nooner, J. L., et al. (2016). Longer interset rest periods enhance muscle strength and hypertrophy in resistance-trained men. J. Strength Cond. Res. 30, 1805-1812. doi: $10.1519 /$ jsc. 0000000000001272

Scudese, E., Simao, R., Senna, G., Vingren, J. L., Willardson, J. M., Baffi, M., et al. (2016). Long rest interval promotes durable testosterone responses in high-intensity bench press. J. Strength Cond. Res. 30, 1275-1286. doi: 10.1519/ JSC.0000000000001237

Scudese, E., Willardson, J. M., Simao, R., Senna, G., de Salles, B. F., and Miranda, H. (2015). The effect of rest interval length on repetition consistency and perceived exertion during near maximal loaded bench press sets. J. Strength Cond. Res. 29, 3079-3083. doi: 10.1097/ JSC. 0000000000000214

Senna, G., Willardson, J. M., de Salles, B. F., Scudese, E., Carneiro, F., Palma, A., et al. (2011). The effect of rest interval length on multi and single-joint exercise performance and perceived exertion. J. Strength Cond. Res. 25, 3157-3162. doi: 10.1519/JSC.0b013e318212e23b

Senna, G. W., Willardson, J. M., Scudese, E., Simao, R., Queiroz, C., Avelar, R., et al. (2016). Effect of different Interset rest intervals on performance of single and multijoint exercises With near-maximal loads. J. Strength Cond. Res. 30, 710-716. doi: 10.1519/JSC.0000000000001142

Shephard, R. J. (1988). PAR-Q, Canadian home fitness test and exercise screening alternatives. Sports Med. 5, 185-195. doi: 10.2165/00007256-198805030-00005 Smith, L. L., Anwar, A., Fragen, M., Rananto, C., Johnson, R., and Holbert, D. (2000). Cytokines and cell adhesion molecules associated with high-intensity eccentric exercise. Eur. J. Appl. Physiol. 82, 61-67. doi: 10.1007/s004210050652

Spiering, B. A., Kraemer, W. J., Anderson, J. M., Armstrong, L. E., Nindl, B. C., Volek, J. S., et al. (2008). Resistance exercise biology: manipulation of resistance exercise programme variables determines the responses of cellular and molecular signalling pathways. Sports Med. 38, 527-540. doi: 10.2165/00007256-200838070-00001

Thomas, D. T., Erdman, K. A., and Burke, L. M. (2016). American college of sports medicine joint position statement. nutrition and athletic performance. Med. Sci. Sports Exerc. 48, 543-568. doi: 10.1249/MSS.0000000000000852

Willardson, J. M., and Burkett, L. N. (2008). The effect of different rest intervals between sets on volume components and strength gains. J. Strength Cond. Res. 22, 146-152. doi: 10.1519/JSC.0b013e31815f912d

Conflict of Interest: The authors declare that the research was conducted in the absence of any commercial or financial relationships that could be construed as a potential conflict of interest.

Publisher's Note: All claims expressed in this article are solely those of the authors and do not necessarily represent those of their affiliated organizations, 
or those of the publisher, the editors and the reviewers. Any product that may be evaluated in this article, or claim that may be made by its manufacturer, is not guaranteed or endorsed by the publisher.

Copyright (c) 2022 Senna, Dantas, Scudese, Brandão, Lira, Baffi, Ribeiro, Simão, Thomas and Bianco. This is an open-access article distributed under the terms of the Creative Commons Attribution License (CC BY). The use, distribution or reproduction in other forums is permitted, provided the original author(s) and the copyright owner(s) are credited and that the original publication in this journal is cited, in accordance with accepted academic practice. No use, distribution or reproduction is permitted which does not comply with these terms. 